\title{
Harlequin Ichthyosis in a HanWoo Calf
}

\author{
Jong-Ki $\mathrm{CHO}^{1)}$, Jung-Min SON ${ }^{1)}$, Doo-Soo LEE ${ }^{1)}$, Seong-Jun PARK ${ }^{1)}$, Sung-Whan $\mathrm{CHO}^{1)}$ and Sang Tae SHIN ${ }^{1) *}$

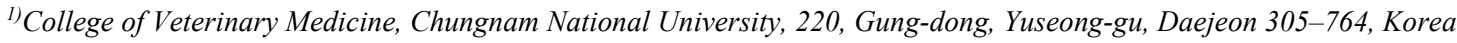

(Received 10 May 2006/Accepted 23 January 2007)

ABSTRACT. Ichthyosis (fish scale disease) is a rare hereditary disease and characterized by excessive cutaneous scale formation. A male HanWoo calf born by natural service was found with fissures and thickened, scaly, cutaneous plates covering over $90 \%$ of its body. Histopathological feature was excess compact orthokeratotic hyperkeratosis involving surface of the epidermis and follicular epithelia. The calf had small malformed ears, ectropion, eclabium and an abnormal nose. Gross and histopathologic findings in calf were consistent with those of harlequin ichthyosis, and it was the first observed harlequin ichthyosis in HanWoo cattle.

KEY WORDS: bovine, HanWoo cattle, harlequin ichthyosis.

Ichthyosis (fish scale disease) is a rare disease of humans, cattle, dogs, pigs, chickens, mice, and llamas and most cases have been reported in calves in the veterinary literature with rare descriptions in the dog $[2,3,5,9-13]$. In cattle, 2 clinical forms of ichthyosis have been recognized and reported. The most severe form is termed Ichthyosis fetalis (Harlequin ichthyosis) and less severe form, termed Ichthyosis congenita is similar to human lamellar ichthyosis $[2,9,12]$. Harlequin ichthyosis, the more severe form of bovine ichthyosis has been described in Norwegian Red Poll, English Friesian, and Swiss breeds $[9,12]$. These calves are stillborn or perish within a few hours or days of birth. Ichthyosis congenita is the milder disorder form than Ichthyosis fetalis and affected calves show variable degrees of generalized hyperkeratosis and alopecia and may be associated with microtia, cataracts and thyroid abnormalities [3, 5, 9-13].

A male HanWoo (Bos taurus coreanae) calf with fissures and thickened, scaly, cutaneous plates covering over $90 \%$ of its body was referred to the Veterinary Medical Teaching Hospital of Chungnam National University. This calf was born by natural service from normal cow and bull. Moreover, the owner used only natural service for breeding without artificial insemination for 20 years and cow and bull were also born by natural service. The calf was small, weighing $19.25 \mathrm{~kg}$ and revealed astasia with tachypnea. Entire body was covered with tight, thick, plate-like scales, with subsequent fissuring. The calf had small malformed ears, ectropion, eclabium, an abnormal nose. Most severely affected areas were skins of the ventral region, inguinal region, face and eyelids. On the basis of history and physical examination, the calf was tentatively diagnosed as a harlequin ichthyosis. Thirteen hours after birth the calf was more depressed and was euthanized due to the severity of the lesions and owner's request.

On the postmortem examination, over $90 \%$ of the calf's body was covered with a $0.5-1 \mathrm{~cm}$ thick dark dry plate-like material and that was partly failing to protrude above the

\footnotetext{
* Correspondence to: Shin, S.T., Laboratory of Theriogenology, College of Veterinary Medicine, Chungnam National University, Gung-dong, Yuseong-gu, Daejeon 305-764, Korea.
}

surface of skin and then fully haired underneath the plate carapace (Fig. 1). Ocular abnormalities included chemosis, erythematous conjunctiva and ectropion. Moreover, the skin around the lips, nose and other orifices tends to be everted and mild varus rotation was observed. In the thorax a patent ductus arteriosus was subsisted. Arthredema was observed in the stifle joint capsule and left testes were located inguinally. All other organs appeared within normal limits.

Tissues from the calf were fixed in $10 \%$ neutral buffered formalin for histopathologic examination and all tissues were stained with hematoxylin and eosin (HE). Histopathologic examination of the epidermis showed marked thick, dense and diffuse hyperkeratosis and partly parakeratosis with no associated inflammation (Fig. 2). The dermis was constituted of mildly thick dense connective tissues. Numerous hair follicles were markedly enlarged and orthokeratosis of dermal root sheath and inner epithelial root sheath was noted up to deep dermis. However, hair shaft appeared histologically normal and was observed in thick stratum corneum. No significant histologic lesions were noted in any other tissues assessed.

The present calf displayed gross and histopathologic findings definitively consistent with harlequin ichthyosis, a group of rare genodermatoses known to break out in variable forms in cattle, dogs, pigs, mice and llamas $[2,3,5,9$ 13].

Ichthyosis is a term used to describe continual scaling of the skin and human ichthyosis is quite rare and can be inherited or acquired $[1,4,6,8]$. The inherited forms of ichthyosis are usually evident at or soon after birth and they tend to persist throughout life although many types improve with age and are classified at least 20 types of ichthyosis [1, 4, 6, $8]$. The most common form of ichthyosis is called ichthyosis vulgaris is inherited in autosomal dominant traits, while rare types of ichthyosis are inherited in autosomal recessive traits $[1,6]$. The most severe form, harlequin ichthyosis is fortunately an extremely rare but causes thick plates of scale and severe complications at birth. The exact incidence is unknown, however it was reported that mutations in the 


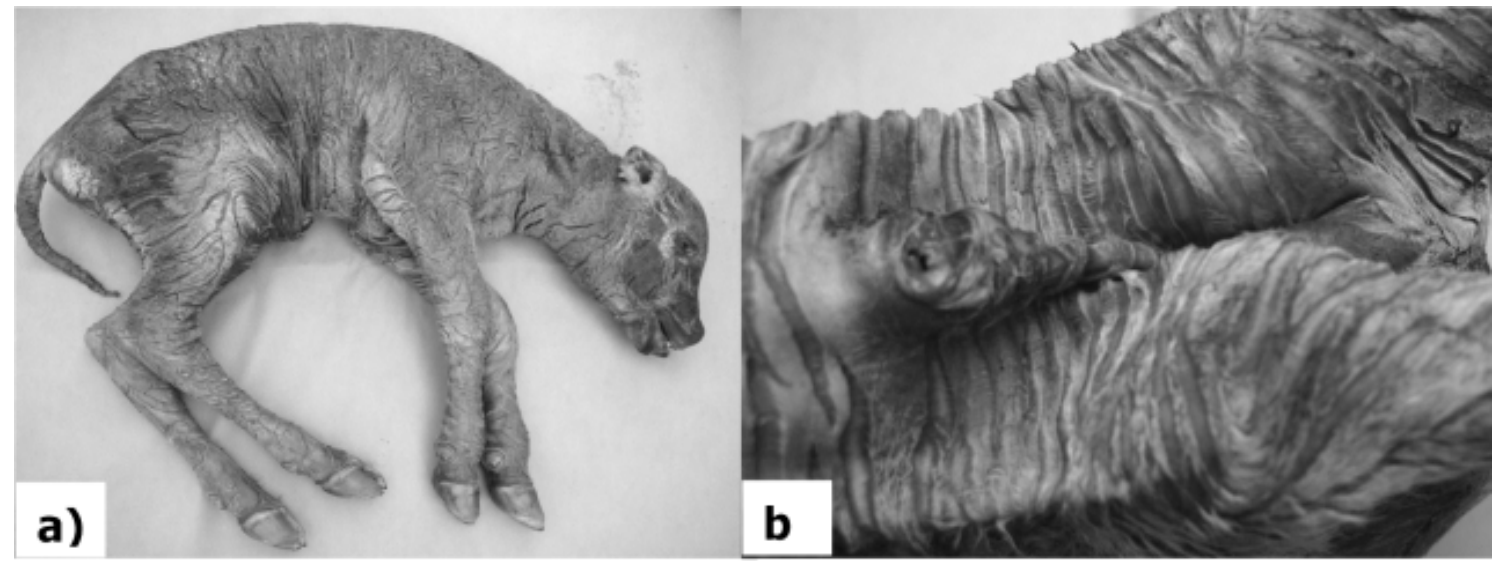

Fig. 1. Hareliquin ichthyosis. a) Whole body of harlequin ichthyosis; Over $90 \%$ of the body is covered with thicken, scaly cutaneous plates and small malformed ears, ectropion, eclabium and a abnormal nose are observed. b) Inguinal region of harlequin ichthyosis; inguinal region is one of most severely affected areas and scaly plates are thicken and deeply fissured.

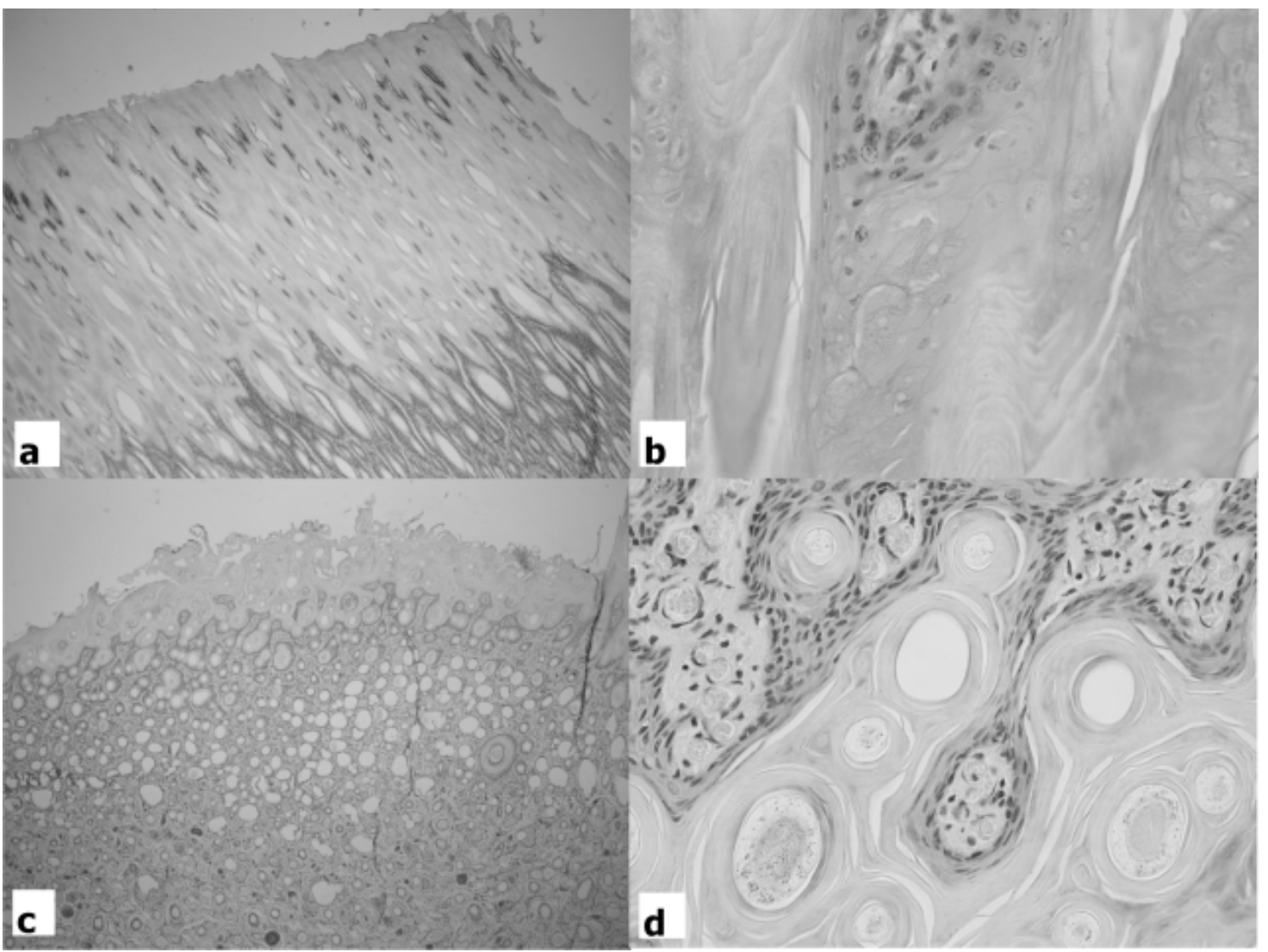

Fig. 2. Histopathological features of the skin from the calf with ichthyosis. Scaly plates were thickened and fissured. Notice marked thick, dense and diffuse hyperkeratosis and there were occasional foci of parakeratosis (HE stain; (a) $\times 40$, (b) $\times 400$ ). Numerous hair follicles were mild dilated (HE stain; (c) $\times 40,(d) \times 400)$.

ABCA12 gene cause harlequin ichthyosis [1]. ABCA12 is a keratinocyte transmembrane lipid transporter protein associated with lipid transport in lamellar granules to the surface of granular layer keratinocytes. In addition, there are other rare types of inherited ichthyoses including with X-linked recessive ichthyosis, non-bullous ichthyosiform erythroderma (lamellar ichthyosis), bullous ichthyosis, Netherton's syndrome, Sjögren-Larsson syndrome and Conradi-Hünermann syndrome $[1,6,8]$.

On the other hand, variable clinical presentations and 
poorly understood histopathogeneses complicate the classification of ichthyosis in veterinary medicine [2, 3, 5, 9-13]. Generally, two congenital forms of ichthyosis are described in cows, ichthyosis congenita and ichthyosis fetalis, and two clinical forms of ichthyosis in cattle are inherited as simple autosomal recessive traits $[2,9,12]$. The skin of a harlequin ichthyosis calf is hairless, or nearly so, and are covered with thick, scaly, horny epithelium that is divided into plates by deep fissures and ichthyosis congenita calves show variable degrees of generalized hyperkeratosis and alopecia and may be associated with microtia, cataracts and thyroid abnormalities [2, 9 11-13].

This calf was born of a normal cow and found with fissures and thickened, scaly, cutaneous plates covering over $90 \%$ of its body. Moreover the calf had small malformed ears, ectropion, eclabium and a abnormal nose. In histopathologic examination, feature was excess compact orthokeratotic hyperkeratosis involving surface of the epidermis and follicular epithelia. In consequence, harlequin ichthyosis was diagnosed on the basis of history, physical examination, gross and histopathologic findings.

In human, various ichthyosis dermatoses have been successfully managed and commercial agents have been developing [4, 6-8]. Generally, neonates were treated with oral drugs, ointments and moisturizing agents to reduce the clinical signs of ichthyosis [7]. Increased humidity is also helpful and supplementary treatments of topical antibiotics like bacitracin are necessary to control pruritus and infection. However, such treatments would appear to be impractical for veterinary medicine [2, 12]. For these reasons, such calves are recommended to euthanasia and the dam and sire are not used for breeding. Moreover, further research is necessary to identify exact causative genes and genetic inspection for the seed bull in the national artificial insemination station.

This case reported the first Harlequin ichthyosis in HanWoo. Harlequin ichthyosis is a very rare genodermatosis and have therefore very little or no importance in the economics of national herds, however it could be financially disastrous to individual owners whose stock are affected [2]. In veterinary medicine, causative genes are exactly unknown, although significant progress has recently been made in the understanding of human ichthyosis. By way of prevention of such a genetic disease, identification of the exact causative genes and genetic inspection for the seed bull are recommended in the national artificial insemination station.

ACKNOWLEDGMENTS. This work was supported by the Research Project on the Production of Bio-organs (No. 200606031401) and High-Technology Development Project (2003-6628) Ministry of Agriculture and Forestry, Republic of Korea.

\section{REFERENCES}

1. Akiyama, M. 2006. J. Dermatol. Sci. (in press).

2. Baker, J.R. and Ward, W.R. 1985. Br. Vet. J. 141: 1-8.

3. Belknap, E.B. and Dunstan, R.W. 1990. J. Am. Vet. Med. Assoc. 197: 764-767.

4. Chan, Y.C., Tay, Y.K., Tan, L.K., Happle, R. and Giam, Y.C. 2003. Pediatr. Dermatol. 20: 421-426.

5. Chittick, E.J., Olivry, T., Dalldorf, F., Wright, J., Dale, B.A. and Wolfe, B.A. 2002. Vet. Pathol. 39: 751-756.

6. Fleckman, P. 2003. Skin. Therapy. Lett. 8: 3-7.

7. Gicquel, J.J., Vabres, P. and Dighiero, P. 2005. J. Fr. Ophtalmol. 28: 412-415.

8. Hazan, C., Orlow, S.J. and Schaffer. J.V. 2005. Dermatol. Online. J. 11: 12.

9. Jones, T.C., Hunt, R.D. and King, N.W. 1997. pp. 827-830. In: Veterinary Pathology. 6th ed., Williams \& Wilkins, Maryland.

10. Olivry, T. and Mason, I.S. 1998. pp. 365-366. In: Advances in Veterinary Dermatology III (Kwochka, K.W., ed.), Butterworth-Heinemann. Boston.

11. Raoofi, A., Mardjanmehr, S.H. and Nekoei, S. 2001. Vet. Rec. 149: 563.

12. Scott, D.W. pp. 339. 1988. In: Large animal dermatology. 1st ed., W.B. Saunders Company. Philadelphia.

13. Scott, D.W., Miller, W.H.J. and Griffin, C.E. 2001. pp. 913 1003. In: Muller \& Kirk's Small Animal Dermatology. 6th ed., W.B. Saunders Company. Philadelphia. 